\title{
PERCEPÇÃO DO IDOSO DOS COMPORTAMENTOS AFETIVOS EXPRESSOS PELA EQUIPE DE ENFERMAGEM
}

\author{
Perception of the elderly in the view of affective behaviors expressed by the Nursing \\ Team \\ Percepción del anciano frente a los comportamientos afectivos expresados por el \\ Equipo de Enfermería
}

Teresa Cristina Prochet ${ }^{1}$

Maria Julia Paes da Silva²

\section{RESUMO}

Objetivo:Identificar a percepção do comportamento da afetividade, pelo idoso hospitalizado, do cuidado recebido pela Equipe de Enfermagem. Material e Método: Estudo quantitativo, transversal e de campo desenvolvido com 28 idosos. Utilizou-se instrumento composto por 21 tipos de comportamento verbais e não verbais. Resultados: Os resultados positivos dos comportamentos verbais incluíram as ações de conversar $(57,2 \%)$, orientar $(60,7 \%)$, respeitar $(50 \%)$, proporcionar segurança $(44,6 \%)$ e demonstrar honestidade $(96,4 \%)$. Os positivos da dimensão não verbal reuniram aspectos relacionados ao respeito $(63,4 \%)$, tocar $(46,4 \%)$, ouvir/escutar $(23,2 \%)$ e olhar como expressão positiva $(71,4 \%)$. Conclusão: A maioria dos idosos percebeu como positiva a afetividade do cuidado recebido pela Equipe de Enfermagem no que se refere à dimensão verbal, sendo 0 comportamento mais evidente a demonstração da sinceridade. As atitudes não verbais de ser ouvido/escutado e tocado com delicadeza assumiram avaliação negativa. A afetividade nas ações de enfermagem são percebidos pelo idoso, e estes interferem na avaliação da qualidade assistencial.

Palavras-chave: Cuidados de enfermagem. Comunicação. Idoso. Enfermagem Geriátrica. Emoções.

\begin{abstract}
Objective: To identify the perception of affective behavior, by the elderly hospitalized, from the care provided by the nursing team. Material and methodology: Quantitative study, cross-sectional and developed with 28 individuals. It was used a tool consisted of 21 types of verbal and nonverbal behavior. Results: The positive results of the actions included verbal behaviors to talk (57.2\%), guide $(60.7 \%)$, respect $(50 \%)$, provide security $(44.6 \%)$ and demonstrate honesty $(96.4 \%)$. The positive of non-verbal dimension joined aspects related to respect (63.4\%), touching (46.4\%), hearing / listening (23.2\%) and the look as a positive expression (71.4\%). Conclusion: Most elderly people perceived as positive the affectivity of care received by the Nursing Team regarding the verbal dimension, with an emphasis on the honesty. The non-verbal attitudes of being heard/listened and touched gently turned into a negative assessment. The affection in nursing actions is perceived by the elderly and these interfere on the assessment of quality of care.
\end{abstract}

Key words: Nursing care. Communication. Aged. Geriatric Nursing. Emotions.

\section{Resumen}

Objetivo: Identificar la percepción de los comportamientos afectivos, por parte del anciano hospitalizado, y del cuidado recibido por los profesionales de enfermería. Metodología: Estudio cuantitativo, transversal y de campo desarrollado con 28 ancianos. Se utilizó un instrumento compuesto por 21 tipos de comportamientos verbales y no verbales. Resultados: Los resultados positivos de los comportamientos verbales incluyeron las acciones de conversar (57,2\%), orientar (60,7\%), respetar (50\%), proporcionar seguridad $(44,6 \%)$ y demostrar honestidad (96,4\%). Los resultados positivos de la dimensión no verbal reunieron aspectos relacionados al respeto $(63,4 \%)$, tocar $(46,4 \%)$, oír/escuchar $(23,2 \%)$ y el miramiento como expresión positiva $(71,4 \%)$. Conclusión: La mayoría de los ancianos percibió como positiva la afectividad del cuidado recibido por los profesionales de enfermería en el que se refiere a la dimensión verbal, siendo el comportamiento más evidente la demostración de sinceridad. Las actitudes no verbales de ser oído/escuchado y tocado con delicadeza asumieron evaluación negativa. La afectividad en las acciones de enfermería es percibida por los ancianos, lo que interfiere en la evaluación de la calidad de la asistencia.

Palabras clave: Atención de Enfermería. Comunicación. Anciano. Enfermería Geriátrica. Emociones.

\footnotetext{
${ }^{1}$ Enfermeira. Doutora em Ciências pela Escola de Enfermagem da Universidade de São Paulo (USP). Integrante do Grupo de Pesquisa e Estudo sobre Comunicação em Enfermagem do CNPq. Docente Comissionada junto ao Departamento Médico Cirúrgico da Escola de Escola de Enfermagem da Universidade de São Paulo. Docente do Departamento de Saúde da Universidade Nove de Julho (UNINOVE). São Paulo - Brasil. E-mail: tcprochet@usp.br; ¿Enfermeira. Profa. Titular da Escola de Enfermagem da Universidade de São Paulo - Departamento Médico Cirúrgico e Coordenadora do Grupo de Estudo e Pesquisa sobre Comunicação em Enfermagem do CNPq. São Paulo-SP. Brasil. E-mail: juliaps@usp.br
} 


\section{INTRODUÇÃO}

0 envelhecimento humano deve ser compreendido pelos profissionais de saúde em toda sua amplitude e totalidade, uma vez que se trata de um crescente fenônemo universal, dinâmico, individual influenciado por fatores biológicos, psicológicos, ambientais, econômicos e sociais. ${ }^{1}$

Apesar de muitos dos idosos serem saudáveis, independentes e capazes de gerir sua própria vida, possuem uma maior susceptibilidade para adoecer do que adultos jovens. Sendo assim, acabam por constituir uma fatia representativa da clientela assistida nos hospitais.

Proporcionalmente, os idosos ocupam mais leitos nos hospitais que os indivíduos de outras faixas etárias e que, realmente, são geradores de gastos maiores. Pode-se questionar se essa ocorrência seria mesmo devido à idade e ao processo de envelhecimento, ou a forma como nosso sistema de saúde lida com a saúde e as doenças das pessoas.?

É sabido que o idoso, quando hospitalizado, requer atenção e cuidados espećficos de forma a colaborar ao máximo na resolutividade dos problemas apresentados, incluindo as limitações próprias relacionadas ao envelhecimento. Para isso é importante que os profissionais da saúde estejam atentos às expectativas dos clientes e compreendam a complexidade e a magnitude dessa etapa vital de maneira a concretizar a essência do cuidado. ${ }^{3} 0$ cuidado gerontológico é um conceito muito ampliado e envolve vários profissionais; nesse artigo, o foco é o cuidado prestado pela Equipe de Enfermagem direcionado ao idoso.

Considera-se neste estudo que o cuidado deva ser uma relação sujeito-sujeito, não devendo ser de domínio sobre o outro, nem tampouco de pura intervenção técnica ${ }^{4}$. Atitudes éticas estão presentes nas ações de cuidar, e a comunicação existente é criada de forma adequada às necessidades de ambos.

A comunicação é um ato criativo que abarca a troca de mensagens e sentimentos entre as pessoas que formam um sistema de interação e reação, ou seja, um processo que provoca mudanças na forma de perceber, sentir, pensar e atuar entre elas. Envolve todos os meios utilizados por uma pessoa para afetar o outro, podendo ser verbal, expressa pelas palavras ditas e escritas, e não verbal, expressa por meio das expressões faciais e gestos corporais, pelo tom, ritmo e entonação das palavras ditas, pela distância mantida entre as pessoas incluindo a forma de aproximação e uso do espaço pessoal e territorial, pelo toque e até pela caracterização e organização do ambiente físico. ${ }^{5}$

Sabe-se que o enfermeiro, ao cuidar do cliente, apresenta comportamentos que são frutos e resultado das experiências que obteve ao longo do tempo em sua prática profissional, sendo alicerçados nos princípios, valores, habilidades e conhecimento. ${ }^{6}$

Quando o cuidado hospitalar é estabelecido de maneira integral e adequada com o idoso, ele permite a criação de vínculo afetivo com os profissionais que cuidam dele. Quando a relação é estreitada, conduz à tranquilidade, segurança e confiança, e o benefício do tratamento é mais facilmente evidenciado. ${ }^{6}$

Essa dimensão afetivo-expressiva faz parte da ação terapêutica do cuidado e pode ser explicitada pela relação de confiança, no trato com carinho, no ser gentil, no demonstrar compreensão, conversar, tocar, falar, escutar, olhar, apoiar, interessar-se, aconselhar entre outros. $^{7}$

Defende-se nesse artigo que afeto é o conjunto de atos ou atitudes como a bondade, a benevolência, a proteção, a gratidão, a ternura, que no seu todo podem ser caracterizados como a situação em que uma pessoa cuida de outra e esta responde positivamente aos cuidados ou à preocupação de que foi objeto. ${ }^{8}$

A prática de enfermagem pode ser caracterizada por ações e comportamentos de cuidar que aliem o espírito científico à emoção, sensibilidade e habilidade técnica. 0 cuidar pode assumir as mais diferentes formas de expressão, revelando também interesse, zelo e valorização do outro. ${ }^{9}$

Para o desenvolvimento desta pesquisa utilizouse do referencial teórico de Waldow ${ }^{6}$ que defende que 0 cuidado é uma ação interativa e como tal resulta no que se define como processo de cuidar. 0 cuidado humano consiste em uma forma de viver, de ser, de se expressar. É um compromisso com o estar no mundo e contribuir para o bem-estar geral, valorizando a dignidade humana e a espiritualidade. É contribuir na construção da história, da vida e do conhecimento. ${ }^{6}$

Nesta perspectiva, é preciso refletir constantemente sobre as implicações físicas, psicoafetivas e comunicacionais que envolvem o cuidado gerontológico, pois quem cuida compartilha os cuidados e não simplesmente pratica uma ação ativamente solitária. Enfermeiros e idosos interagem durante a prestação do cuidado, juntos promovem um processo contínuo de criação e reprodução dos sentidos, mesmo que, nem sempre, de maneira consciente.

Investigar como os idosos hospitalizados percebem o cuidado prestado pelo enfermeiro e sua equipe representa uma contribuição na busca da qualidade da assistência de enfermagem. Além disso, vai ao encontro dos pressupostos da ética profissional, tão almejados pelos enfermeiros e demais profissionais de saúde envolvidos na área de geriatria e gerontologia.

\section{OBJ ETIVO}

Identificar a percepção de comportamentos verbais e não verbais da afetividade, pelo idoso hospitalizado, do cuidado recebido pela Equipe de Enfermagem. 


\section{MATERIAIS E MÉTODOS}

Estudo quantitativo e transversal desenvolvido em um hospital público do interior paulista após parecer favorável do Comitê de Ética em Pesquisa com Seres Humanos (CEP-HRA n $52 / 2008)$. Trata-se de uma amostra intencional com 28 pessoas de ambos os sexos com idade igual ou superior a 60 anos. Este número baseou-se em $50 \%$ da taxa média mensal de internação de idosos nas clínicas médica e cirúrgica da instituição. Critérios de inclusão: ter sido internado há no mínimo 72 horas, nas unidades de clínica médica e cirúrgica; não apresentar declínio cognitivo conforme resultado obtido pelo miniexame do estado mental de Folstein. ${ }^{10}$

Os idosos foram submetidos ao índice de dependência proposto por Waldow ${ }^{11} \mathrm{e}$ a dois questionários: um para identificar o perfil sociodemográfico e outro denominado Identificação da Percepção do Cuidado pelo Paciente (IPCP), validado por Bobroff'12, que consta de 21 comportamentos do cuidado. Cada idoso respondeu o que sentiu, percebeu ou compreendeu imediatamente após ter recebido algum tipo de cuidado pela Equipe de Enfermagem, tendo três opções de resposta (sim, um pouco e não).

0 tratamento dos dados foi feito por meio de análise descritiva, utilizando-se a divisão proposta ${ }^{12}$ que incorpora os comportamentos observáveis nas dimensões verbal e não verbal. A comunicação verbal constou de 13 perguntas relativas a conversar, orientar, respeitar, proporcionar segurança e demonstrar honestidade/sinceridade. 0 não verbal englobou oito perguntas focadas no respeitar, tocar, ouvir/escutar e olhar.

\section{RESULTADOS}

Todos os idosos pesquisados eram brasileiros, tinham idade média de 70,8 anos, 60,7\% eram do sexo feminino, com 3,5 anos, em média, de estudo; 92,8\% professaram algum tipo de religião, a católica representada por 76,9\% e a evangélica com 23,1\%. Em relação ao estado civil, $57,1 \%$ dos idosos estavam casados, $21,4 \%$ viúvos, $17,9 \%$ solteiros e $3,6 \%$ divorciados. A renda média foi de 1,4 salários mínimos. Apesar de 71,4\% informarem estar aposentados, $20 \%$ desses continuam exercendo alguma atividade trabalhista como forma de melhorar a renda familiar.

No momento da aplicação do questionário a média de internação dos idosos era de 9,4 dias. 0 grau de dependência física apresentou os resultados de: 60,7\% independentes e $39,3 \%$ de dependentes do grau I.

A seguir constam as frequências das respostas sobre os comportamentos verbais (Tabela 1) e não verbais (Tabela 2) expressos pelos idosos diante da Equipe de Enfermagem.

Tabela 1.Distribuição de frequência das respostas dos 28 idosos sobre os comportamentos verbais observáveis do cuidado prestado pela Equipe de Enfermagem, Assis - 2009.

\begin{tabular}{lccc}
\hline Comportamentos Verbais do Cuidado & \multicolumn{2}{c}{ Respostas do Idoso } \\
\hline 1. Conversar & Sim & Não \\
Cumprimento ao idoso & $21(75.0)$ & $07(25.0)$ \\
Apresentação ao idoso & $11(39.3)$ & $17(60.7)$ \\
2. Orientar & Sim & Um pouco & Não \\
Orientação ao idoso sobre o cuidado a ser prestado & $17(60.7)$ & $03(10.7)$ & $08(28.6)$ \\
\hline 3. Respeitar & Sim & Um pouco & Não \\
Autorização para tocar o idoso & $10(35.7)$ & $00(0.0)$ & $18(64.3)$ \\
Respeito pela vontade do idoso sem colocá-lo em risco & $23(82.2)$ & $05(17.8)$ & $00(0.0)$ \\
Estímulo da participação do idoso no cuidado & $09(32.1)$ & $05(17.8)$ & $14(50.0)$ \\
\hline 4. Proporcionar segurança & Sim & Um pouco & Não \\
Conversa com o idoso durante a prestação do cuidado & $15(53.6)$ & $05(17.8)$ & $08(28.6)$ \\
\hline Tranquiliza o idoso & $16(57.1)$ & $04(14.3)$ & $08(28.6)$ \\
Demonstração ao idoso de firmeza e segurança & $27(96.4)$ & $01(3.6)$ & $00(0.0)$ \\
Conversa com o idoso sobre assuntos do interesse dele & $09(32.1)$ & $03(10.7)$ & $16(57.1)$ \\
Explicação ao idoso sobre o motivo da intemação & $03(10.7)$ & $03(10.7)$ & $22(78.6)$ \\
Ação do cuidado ao idoso com pressa & $05(17.8)$ & $04(14.3)$ & $19(67.9)$ \\
\hline 5. Demonstrar sinceridade/honestidade & Sim & Um pouco & Não \\
Parece ser sincera com o idoso & $27(96.4)$ & $01(3.6)$ & $00(0.0)$ \\
\hline
\end{tabular}

Nota: ${ }^{\circ}{ }^{\circ}=$ valor numérico relativo; $(\%)=$ valor numérico percentual. 
Tabela 2 .Distribuição de frequência das respostas dos 28 idosos sobre os comportamentos não verbais observáveis do cuidado prestado pela Equipe de Enfermagem, Assis - 2009.

\begin{tabular}{lccc}
\hline Comportamentos Não Verbais do Cuidado & \multicolumn{3}{c}{ Respostas do Idoso } \\
\hline 1. Respeitar & Sim & Um pouco & Não \\
Respeito aos sentimentos do idoso & $20(71.4)$ & $08(28.6)$ & $00(0.0)$ \\
Aceitação do idoso & $15(53.6)$ & $13(46.4)$ & $00(0.0)$ \\
Respeito pela não exposição do idoso durante o cuidado & $08(28.6)$ & $05(17.8)$ & $15(53.6)$ \\
Acredita no idoso & $28(100.0)$ & $00(0.0)$ & $00(0.0)$ \\
2. Tocar & Sim & Um pouco & Não \\
Toque no idoso com delicadeza & $13(46.4)$ & $10(35.7)$ & $05(17.9)$ \\
3. Ouvir/Escutar & Sim & Um pouco & Não \\
Escuta o idoso com atenção e aceitação & $08(28.6)$ & $12(42.9)$ & $08(28.6)$ \\
Silencia para escutar o idoso & $05(17.9)$ & $01(3.6)$ & $22(78.6)$ \\
4. Olhar & Sim & Um pouco & Não \\
Olhar ao idoso com expressão positiva & $20(71.4)$ & $08(28.6)$ & $00(0.0)$ \\
\hline
\end{tabular}

Nota: $\mathrm{N}^{\circ}=$ valor numérico relativo; $(\%)=$ valor numérico percentual

\section{DISCUSSÃO}

A comunicação é uma necessidade humana básica e, portanto, engloba os aspectos verbais e não verbais envolvidos na assistência ao cliente. ${ }^{5}$ Conforme já referido, o profissional de saúde responsável e comprometido com o cuidado tem o dever de se apropriar do conhecimento e desenvolver habilidades tanto técnicas procedimentais como de comunicação interpessoal.

Verificou-se que o comportamento verbal do cuidado fornecido pela equipe de enfermagem (Tabela 1), no que se refere às atitudes de conversar com ele, foi positivamente percebida pelo cumprimento dado pelo profissional antes de prestar-lhe um cuidado; entretanto, a apresentação pessoal foi negligenciada por grande parte da enfermagem. 0 fato de 0 idoso não reconhecer a apresentação do profissional de enfermagem responsável pelo cuidado pode explicitar o esquecimento da equipe de saúde na informação dos seus direitos, desde um dos mais básicos, que é o de conhecer quem o trata. Parece existir atualmente a necessidade de mudança na atitude do profissional, para que uma relação empática e participativa seja realmente construída com o cliente e, assim, ele saiba reconhecer não só quem são os profissionais, mas também quais são as suas responsabilidades e seus direitos. ${ }^{13}$

Os resultados revelaram que $60,7 \%$ dos idosos informaram terem sido orientados, pela enfermagem, sobre o cuidado que foi prestado. Isso vai ao encontro do estudo feito com idosos hospitalizados que detectou que explicar o que e como será feita uma determinada atividade é uma atitude percebida como favorável ao estabelecimento de um atendimento humanizado, que desvela cuidado. ${ }^{13}$

Apesar de $64,3 \%$ dos idosos serem tocados pela equipe de enfermagem sem sua autorização prévia, $82,2 \%$ perceberam que a atitude desses profissionais, durante a execução da tarefa, respeitou sua vontade não o deixando correr risco e 50\% consideraram que foram estimulados à participação do cuidado. Aqui se pode verificar o quanto o profissional de saúde negligencia, na prática, o direito básico do cliente que é o de dar o consentimento para algo, antes que este seja realizado., ${ }^{5,13}$ A participação do idoso no cuidado, conforme já referido, constitui uma atitude de zelo e de atenção, além de favorecer que o vínculo afetivo seja estabelecido e mantido., ${ }^{6,13}$

A capacidade de proporcionar segurança ao idoso foi valorizada por ele quando a equipe de enfermagem, durante a prestação do cuidado, conversou com idoso $(53,6 \%)$, tranquilizando-o (57,1\%). A demonstração ao idoso de firmeza e segurança técnica foi o item que mais se destacou, atingindo $94,6 \%$ das respostas. Entretanto, os profissionais de enfermagem acabaram por não explicar o motivo da internação $(78,6 \%)$, realizaram o cuidado com pressa $(67,9 \%)$ e não revelaram interesse em conversar sobre outros assuntos do interesse do idoso $(57,1 \%)$, mesmo sabendo que quando a pessoa é internada passa a viver em um ambiente de impessoalidade, onde o temor pelo desconhecido, a frieza dos procedimentos levam às fragilidades e preocupações. Estudo desenvolvido com idosos hospitalizados em São Paulo ${ }^{14}$ constatou que raramente o cliente é informado das suas condições de saúde e do motivo que gerou sua internação e, ainda, mal sabe o nome do medicamento que recebe, não participando das decisões de sua vida e se tornando vulnerável ao cuidado eminentemente técnico.

É essencial que seja proporcionado ao idoso um cuidado técnico de qualidade e que Ihe traga segurança. Idosos internados em clínica médica citam quanto 0 valorizam 0 procedimento técnico, demonstrando como as ações de 
cumprimento de ordens médicas, prazos e horários corretos e a revelação da destreza manual do profissional constituem exemplos evidenciados que trazem segurança. Contudo, o profissional, ao utilizar linguagem técnica, acaba por excluir a participação do idoso no processo de cuidar, provocando nele sentimentos de medo, apreensão e insegurança. ${ }^{13}$

O uso do tempo da execução das técnicas para conversar sobre assunto de interesse do cliente é reconhecido como uma atitude de aproximação, é sinal de que o profissional exerce empatia, demonstra interesse pelo outro, resultando em sentimento de segurança. ${ }^{5,13}$

Foi unânime entre os idosos a percepção de que a Equipe de Enfermagem, durante a execução dos procedimentos, demonstrou sinceridade e honestidade, o que é positivo sempre. 0 resultado vai ao encontro do Código de Ética dos Profissionais de Enfermagem, que exige, dentre outras responsabilidades e deveres, que os profissionais exerçam suas atividades com justiça, compromisso, equidade, resolutividade, dignidade, competência, responsabilidade, honestidade e lealdade, independentemente do local de trabalho. ${ }^{15}$

Não há dúvida de que as unidades de internação são espaços de comunicação e que as dimensões verbais e não verbais orientam as relações entre os idosos internados e a equipe de enfermagem, permitindo uma constelação de mensagens que podem ser captadas e decodificadas de forma consciente ou inconsciente.

Como pode ser percebido na Tabela 2, várias dimensões do não verbal foram avaliadas. Como resultado, os aspectos ligados ao respeito pelos sentimentos do idoso $(71,4 \%)$, incluindo o acreditar da equipe de enfermagem diante das expressões vocais e não vocais (100\%), foram citados pelos participantes do estudo. Constituem como funções da comunicação não verbal a demonstração de sentimentos; complementação, ratificação e reforço do verbal; contradição ou substituição do verbal. A comunicação não verbal desvenda a essência do indivíduo, de suas emoções e pensamentos, potencializando a comunicação do profissional com o cliente e equipe Observar o não verbal de si e do outro, ser sensível às necessidades do outro, utilizar cuidadosamente as palavras e acreditar no relato das pessoas são fatores que contribuem e influenciam para que a comunicação seja efetiva e afetiva. ${ }^{5}$

As dimensões próprias da comunicação não verbal, como a tacêsica (tocar), paralinguagem (ouvir e escutar), cinésica (olhar) e proxêmica (violação do espaço pessoal), também foram investigadas. A maioria dos idosos respondeu que o toque exercido pela equipe de enfermagem não foi com delicadeza (53,6\%). 0 toque é considerado uma das maneiras mais importantes de comunicação não verbal. É sabido que, dependendo do momento, forma e local onde ocorre, envia mensagens positivas ou negativas ao cliente. A análise do toque deve constar das variáveis como: duração, localização (se ocorre em áreas mais sensíveis, mais externas ou mais próximas do coração), velocidade de aproximação do outro (abrupta ou gradual), intensidade ou a pressão exercida no outro, frequência e sensação provocada (reação de conforto ou desconforto na pele ao receber ou transmitir o estímulo). Quando se conhece esses quesitos, possibilitam-se condições nas quais o toque seja exercido de maneira eficiente. .,16 $^{5}$

0 fato de o idoso não perceber delicadeza durante 0 toque remete à pesquisa na qual se verificou que a experiência tacêsica durante a realização de procedimento técnico é considerada a mais marcante para as expressões emotivas do cliente. Ficou evidenciado que, por meio do toque, a enfermagem transmite mais concretamente os sentimentos de empatia e confiança. ${ }^{16}$

A minoria dos pesquisados $(28,6 \%)$ percebeu de maneira positiva que a escuta com atenção foi realizada pelos profissionais, e 78,6\% evidenciou que a enfermagem não silenciou para escutá-lo. Esses resultados vão ao encontro da afirmativa na literatura que defende que a tendência de fragmentar o que se ouve é maior do que a integração do que se fala e escuta. É comum os profissionais não escutarem até o fim da fala, comparando, quase que imediatamente, a mensagem que o outro tenta transmitir com ideias e referenciais prévios. ${ }^{5} \mathrm{Na}$ verdade isto é resultado de um condicionamento social e mental denominado de automatismo concordo-discordo. Em outras palavras, quando o cliente fala, o profissional acaba assumindo duas atitudes: a primeira é de que ele já sabe 0 que será dito e concorda logo, para não "perder tempo", ouvindo-o; a segunda é de que ele também sabe o que será dito e discorda, assim não precisa ouvi-lo até o fim. Em ambos os casos, se nega ao cliente a capacidade ou a possibilidade de contar algo novo e importante.

Para ser efetivo/afetivo na comunicação, é necessário estar disposto não só a escutar o outro, mas olhar para o outro. 0 olhar compõe juntamente com gestos e movimentos corporais a cinésica. ${ }^{5}$ Dos idosos estudados, $71,4 \%$ se sentiram olhados de maneira positiva, o que está ratificado na pesquisa realizada que identificou o quanto que o olhar e as expressões faciais das pessoas que cuidam geram segurança, confiança e simpatia aos pacientes. ${ }^{17}$ Considerando que a gestualidade, expressão facial e olhares interferem no resultado do cuidado, torna-se essencial que a equipe de enfermagem valorize e execute suas atividades de uma maneira mais consciente. Dependendo do jeito, duração e para onde se olha o cliente, o cuidado afetivo é percebido ou não. ${ }^{15}$

A proxêmica estuda o significado social do espaço, ou seja, analisa como o homem estrutura consciente ou inconscientemente o próprio espaço, incluindo tanto as especificidades do espaço pessoal como a territorialidade. ${ }^{5}$ Neste estudo, o respeito pela não exposição corporal do idoso durante o cuidado foi considerado negligenciado por $53,6 \%$ dos idosos estudados. 0 resultado remete à pesquisa que verificou que as reações de desagrado dos 
idosos hospitalizados diante das situaç̃es que caracterizam invasão de seu espaço pessoal foram aquelas ligadas à exposição de partes íntimas durante a realização dos procedimentos. $^{18}$

\section{CONSIDERAÇÕES FINAIS}

De acordo como objetivo proposto, verificou-se que a percepção da afetividade pelos idosos hospitalizados do cuidado recebido pela equipe de enfermagem foi mais positiva nos comportamentos verbais que os não verbais. 0 comportamento verbal que recebeu maior percentual de satisfação foi a demonstração da sinceridade e honestidade durante a prestação do cuidado; o menor foi atribuído à segurança proporcionada, principalmente no sentido de não ter sido dada a ele explicação sobre o motivo de sua internação e pela execução do cuidado ter sido realizado de maneira apressada. Em relação ao comportamento não verbal, identificou-se que o mais satisfatório foi a forma de olhar com expressão positiva ao idoso, e o negativo, a atitude da enfermagem em não ouvi-lo com atenção e interesse, bem como de não silenciar para escutá-lo.

As recentes transformações tecnológicas e científicas na área da saúde trouxeram inúmeros benefícios sociais e profissionais; porém, isso não garante qualidade na assistência prestada ao cliente, visto as múltiplas variáveis existentes. Sem dúvida, consideramos que o afeto compõe, juntamente com outros quesitos, a busca pela concretização do cuidado integralizado e humanizado que toda pessoa necessita. A afetividade possui um conteúdo psíquico que dá "cor e movimento" aos pensamentos, motivações, temperamentos e outros mais, alterando substancialmente o estado de ânimo das pessoas e interferindo positivamente na recuperação de quem esteja vivenciando situações difíceis, como é o caso da internação hospitalar.

Conhecer o que os idosos observam, pensam e sentem proporciona oportunidade não só para reflexão da prática da equipe de enfermagem, mas também permite que medidas reais sejam tomadas e fortaleçam o vínculo de quem cuida e de quem é cuidado. Defendemos que o fazer em enfermagem não deva ser voltado exclusivamente ao cumprimento da tarefa, mas sim, que o cuidado pode e deve ser planejado e promovido em parceria com o idoso, valorizando suas peculiaridades e limitações.

Este estudo avança no conhecimento da área de Enfermagem, pois traz contribuiç̃̃es sobre a importância e a construção das competências do saber-conviver no âmbito da Enfermagem Gerontológica, bem como revela que o tempo efetivo/afetivo no cuidado com qualidade é uma atitude observada e valorizada pelos idosos durante a prestação do atendimento, sendo necessária para que as relações interpessoais sejam adequadamente estabelecidas e mantidas.

\section{REFERÊNCIAS}

1.Organização Mundial de Saúde- OMS. Envelhecimento ativo: uma política de saúde. Tradução de S Gontijo. Brasília (DF); 2005.

2.Alvarenga MRM, Mendes MMR. 0 perfil das readmissões de idosos num hospital geral de Marilia/SP. Rev Latino-am Enfermagem. 2003 jun.; 11 (3): 305-11.

3.Martins JJ, Barra DCC, Souza EA, Nostrami ZMO, Nascimento ERP, Erdmann $A L$, et al. Influência do processo de envelhecimento na qualidade de vida do ser humano: (re)desvelando significados. REME Rev Min Enferm. 2007 jul./set.; 11(3): 265-71.

4. Ribeiro MIC, Furegato ARF. Reflexões sobre a importância do relacionamento interpessoal na formação de profissionais de enfermagem. Nursing, São Paulo. 2003 nov; 6(66):19-24.

5.Silva MIP. Comunicação tem remédio: a comunicação nas relações interpessoais em saúde. São Paulo: Loyola; 2002.

6.Waldow VR. Cuidado humano: o resgate necessário. $3^{\mathrm{a}}$ ed. Porto Alegre: Sagra Luzzatto; 2001.

7.Gonzaga MLC, Arruda EM. Fontes e significados de cuidar e não cuidar em hospital pediátrico. Rev Latino-am Enfermagem. 1998 dez; 6(5):1726.

8.Monte-Serrat F. Emoção, afeto e amor: ingredientes do processo educativo. São Paulo: Academia de Inteligência; 2007.

9.Silva AL. 0 cuidado no encontro de quem cuida e de quem é cuidado. In: Meyer DE, Waldow VR, Lopes MJM, organizadores. Marcas da diversidade: saberes e fazeres da enfermagem contemporânea. Porto Alegre: Artmed; 1998. p. 195.

10.Folstein MF, Folstein SE, McHugh PR. Mini-mental state: a practical method for grading the cognitive state of patients for the clinician. J Psychiatry Res. 1975 Nov; 12(03): 189-98.

11.Waldow VR. Determinação do nível de dependência em enfermagem junto a idosos institucionalizados de Porto Alegre. Rev Gaucha Enferm. 1987 jan; 8(1): 46-73.

12.Bobroff MCC. Identificação de comportamentos de cuidado afetivoexpressivos no aluno de enfermagem: construção de instrumentos [dissertação]. Ribeirão Preto (SP): Escola de Enfermagem de Ribeirão Preto, Universidade de São Paulo; 2003.

13.Prochet TC, Ruiz T, Correa I. A humanização do atendimento ao idoso: o que o idoso hospitalizado sente, percebe e deseja? Nursing,São Paulo. 2006 mar; 9(94): 713-18.

14.Conselho Regional de Enfermagem-COREN-SP. Principais legislaç̃̃es para o exercício da enfermagem. São Paulo; 2008.

15.Prochet TC. Capacitação em comunicação não verbal: um caminho para ações de cuidado efetivo/afetivo ao idoso [tese]. São Paulo: Escola de Enfermagem, Universidade de São Paulo; 2010. 
16.Araujo STC, Moura VLF, Sória DAC, Lima EMS. A semiologia das expressões dos pacientes com feridas cirúrgicas no pós-operatório imediato. Esc Anna Nery. 2004 abr; 8(1): 53-61.

17.Prochet TC, Silva MJP. Competência comunicacional: o que é isso? RECENF: revista tecnico- cientifica de enfermermagem. 2010 jul./ago; 8(25): 118-24.

18.Prochet TC, Silva MJP. Situações de desconforto vivenciadas pelo idoso hospitalizado com a invasão do espaço pessoal e territorial. Esc Anna Nery. 2008 jun; 12(2): 310-15. 